\title{
CEACAM6 gene expression in intrahepatic cholangiocarcinoma
}

\author{
K leta',2, F Tanaka', T Utsunomiya', H Kuwano² and M Mori", ${ }^{*}$ \\ 'Department of Surgery and Molecular Oncology, Medical Institute of Bioregulation, Kyushu University, 4546 Tsurumihara, Beppu, 874-0838, Japan; \\ ${ }^{2}$ Department of General Surgical Science, Graduate School of Medicine, Gunma University, 3-39-22 Showa-machi, Maebashi, Gunma, 37 I-85 I I, Japan
}

The purpose of this study was to investigate the clinicopathological and biological significance of human carcinoembryonic antigenrelated cell adhesion molecule 6 (CEACAM6) gene expression in human intrahepatic cholangiocarcinoma. CEACAM6 is reported to be involved in human malignancies. However, in cholangiocarcinoma expression of CEACAM6 and its clinicopathological significance have not been investigated. CEACAM6 expression status was determined and analysed with respect to various clinicopathological parameters in 23 intrahepatic cholangiocarcinomas. Additionally, we investigated effects of CEACAM 6 gene in the cholangiocarcinoma cell lines. CEACAM6 gene expression in cancer tissues was higher than in noncancerous tissues in 16 of the 23 cases; however, it was not statistically significant. The tumours with elevated CEACAM6 expression showed a tendency to be associated with lymphatic invasion and stage of the disease. Interestingly, patients with high CEACAM6 expression showed a significantly poorer disease-free survival rate than those with low CEACAM6 expression. We demonstrated that CEACAM6-transfected cells were more proliferative, more invasive and more chemoresistant to gemcitabine compared to mock-transfected cells. Furthermore, CEACAM6 gene silencing by CEACAM6-specific siRNA resulted in higher chemosensitivity to gemcitabine. CEACAM6 is a potential prognostic indicator and potential chemoresistant marker to gemcitabine for patients with intrahepatic cholangiocarcinoma.

British Journal of Cancer (2006) 95, 532-540. doi:I0.I038/sj.bjc.6603276 www.bjcancer.com

Published online 25 July 2006

(C) 2006 Cancer Research UK

Keywords: carcinoembryonic antigen-related cell adhesion molecule 6 (CEACAM6); cholangiocarcinoma; gemcitabine

Cholangiocarcinoma is a malignant neoplasm arising from the biliary epithelium, either within the liver (intrahepatic cholangiocarcinoma) or extrahepatic bile duct (extrahepatic cholangiocarcinoma). Although it is a relatively rare malignancy, the worldwide incidence of intrahepatic cholangiocarcinoma is steadily increasing (Olnes and Erlich, 2004; Khan et al, 2005; Lazaridis and Gores, $2005)$. The overall survival rate is poor; $<5 \%$ of patients survive more than 5 years, and the rate has not changed significantly over the past 30 years (Shaib and El-Serag, 2004). The curative treatment of cholangiocarcinoma is surgical resection. More than two-thirds of patients with cholangiocarcinoma are not resectable at the time of initial diagnosis (Khan et al, 2002), because it is clinically silent until it has become an advanced disease with obstructive symptoms. Chemotherapy for cholangiocarcinoma is carried out for those patients who are inoperable or who have recurrent disease, but the results tend to be disappointing (Olnes and Erlich, 2004; Khan et al, 2005). Several different new anticancer drugs are under investigation for the treatment of advanced cholangiocarcinoma (Thongprasert, 2005). Among those, one agent - the nucleoside analog gemcitabine - was reported to show efficacy in treating advanced cholangiocarcinoma (Thongprasert, 2005).

Carcinoembryonic antigen-related cell adhesion molecule 6 (CEACAM6) is a glycosylphosphatidylinositol(GPI)-linked immunoglobulin super family member (Thompson et al, 1991) that is

* Correspondence: Professor M Mori;

E-mail: mmori@beppu.kyushu-u.ac.jp

Received I February 2006; revised 2 June 2006; accepted 22 June 2006; published online 25 July 2006 overexpressed in a variety of malignancies (Hasegawa et al, 1993; Kodera et al, 1993; Jantscheff et al, 2003; Duxbury et al, 2004a). Despite lacking an intracellular domain, CEACAM6 is able to influence intracellular signaling events, and overexpression of this molecule appears to promote gastrointestinal cancer progression (Scholzel et al, 2000; Ilantzis et al, 2002). Silencing of the CEACAM6 gene impairs metastasis and suppresses tumor growth (Duxbury et al, 2004a,b). In cholangiocarcinoma, CEACAM6 expression and its relation to clinicopathological factors have not been investigated.

In the present study, we investigated the expression of CEACAM6 in patients with intrahepatic cholangiocarcinoma using real-time quantitative reverse transcription-polymerase chain reaction (RT-PCR) to analyse the association of clinicopathological factors and prognosis with CEACAM6 expression levels. Furthermore, we established a CEACAM6 stably transfected human cholangiocarcinoma cell line and examined the biological behaviour of CEACAM6-transfected cells, such as cell growth, invasiveness, resistance to anoikis and gemcitabine chemosensitivity. We also examined whether silencing the CEACAM6 gene by siRNA enhanced gemcitabine chemosensitivity.

\section{MATERIALS AND METHODS}

\section{Cell lines}

The human cholangiocarcinoma cell lines, TFK-1, HuCC-T1 and MEC were provided by the Cell Resource Center of Biomedical 
Research, Institute of Development, Aging and Cancer (Tohoku University, Sendai, Japan) and maintained in RPMI 1640 medium containing $10 \%$ fetal bovine serum (FBS) and antibiotics at $37^{\circ} \mathrm{C}$ in a $5 \%$ humidified $\mathrm{CO}_{2}$ atmosphere.

\section{Clinical samples}

Patients (23) with intrahepatic cholangiocarcinoma who underwent surgery at the Medical Institute of Bioregulation Hospital and the Department of Surgery and Science, Kyusyu University between 1993 and 2002 were enrolled in this study. All patients underwent a resection of the primary cancer. No patients received chemotherapy or radiotherapy prior to or after operation. The resected cancer $(\mathrm{T})$ and paired noncancerous $(\mathrm{N})$ tissue specimens were immediately frozen in liquid nitrogen and kept at $-80^{\circ} \mathrm{C}$ until the extraction of RNA. Written informed consent was obtained from all patients. The follow-up ranged from 1 to 74 months with a median of 12 months.

\section{Oligonucleotide primers for CEACAM6 gene amplification by RT - PCR}

Total RNA was extracted from each sample and complementary DNA (cDNA) was synthesized from $8 \mu \mathrm{g}$ of total RNA using random hexamer primers and M-MLV reverse transcriptase (Invitrogen Crop., Carlsbad, CA, USA) as described previously (Mori et al, 2002).

The oligoribonucleotide primers for CEACAM6 (226bp) were sense primer: 5'-GAAATACAGAACCCAGCGAGTGC-3'; antisense primer: $5^{\prime}$-CAGTGATGTTGGGGATAAAGAGC-3', glyceraldehyde 3-phosphate dehydrogenase $(G A P D H)(270 \mathrm{bp})$ sense primer: $5^{\prime}$ TTGGTATCGTGGAAGGACTCA-3'; antisense primer: $5^{\prime}$-TGTCA TCATATTTGGCAGGTT- $3^{\prime}$. To avoid amplification of contaminating genomic DNA, these primers spanned more than two exons. The amplification was performed for 30 cycles of $1 \mathrm{~min}$ at $95^{\circ} \mathrm{C}$, $1 \mathrm{~min}$ at $69^{\circ} \mathrm{C}$ and $1 \mathrm{~min}$ at $72^{\circ} \mathrm{C}$. An $8 \mu \mathrm{l}$ aliquot of each PCRamplified DNA was electrophoresed on a $2 \%$ agarose gel containing ethidium bromide. To ensure that the RNA was of sufficient purity for RT-PCR, a PCR assay with primers specific for the GAPDH gene was carried out in each case, except that only 22 cycles were performed under the following cycling conditions: $1 \mathrm{~min}$ at $95^{\circ} \mathrm{C}, 1 \mathrm{~min}$ at $56^{\circ} \mathrm{C}$ and $1 \mathrm{~min}$ at $72^{\circ} \mathrm{C}$.

\section{Real-time quantitative RT - PCR}

The PCR amplification for quantification of CEACAM6 and GAPDH mRNA in clinical samples was performed in the LightCycler system (Roche Applied Science, IN, USA) using the LightCycler-FastStart DNA Master SYBR Green I kit (Roche Applied Science, IN, USA) as described previously (Ogawa et al, 2005). The amplification conditions of cycles consisted of initial denaturation at $95^{\circ} \mathrm{C}$ for $10 \mathrm{~min}$, followed by 40 cycles of denaturation at $95^{\circ} \mathrm{C}$ for $10 \mathrm{~s}$, annealing at $72^{\circ} \mathrm{C}\left(60^{\circ} \mathrm{C}\right.$ for $\left.G A P D H\right)$ for $10 \mathrm{~s}$, and elongation at $72^{\circ} \mathrm{C}$ for $10 \mathrm{~s}$. For distinguishing specific from nonspecific products and primer dimers, melting curve analysis was carried out. To verify the melting curve results, each representative sample of the PCR products was electrophoresed on $2 \%$ agarose gels, and a single PCR product of the size predicted from the cDNA was confirmed. To quantitate the amount of specific mRNA in the samples, a standard curve was produced for each run measuring three points of the diluted TFK-1 cDNA. The concentrations of each sample were calculated by observing their crossing point to a standard curve. The relative expression levels of CEACAM6 were obtained by normalizing the amount of CEACAM6 mRNA divided by that of GAPDH mRNA as an endogenous control in each sample. Each assay was performed at least twice to verify the results, and the mean mRNA expression was used for analysis.

\section{Immunohistochemistry}

Immunohistochemical studies of CEACAM6 were performed on surgical specimens from intrahepatic cholangiocarcinoma patients on formalin-fixed, paraffin-embedded tissues. After deparaffinization and blocking, the antigen-antibody reaction was incubated overnight at $4{ }^{\circ} \mathrm{C}$. ENVISION reagents (ENVISION + Dual Link/ HRP, Dako Cytomation, Denmark) were applied to detect the signal of the antigen-antibody reaction. All sections were counterstained with haematoxylin. The primary mouse monoclonal antibodies against CEACAM6 (GM7G5, Alexis Biochemicals, USA) were used at dilution of $1: 100$.

\section{Flow cytometry analysis}

The cells were harvested and rinsed twice with PBS. Dissociated cells were stained with PE-conjugated anti-CEACAM6 antibody (KOR-SA3544 antigen-PE, Beckman Coulter, USA) and incubated for $30 \mathrm{~min}$ at room temperature. Cells $(10000)$ were collected for each sample using FACScan, and the data were analysed with CellQuest software (Becton Dikinson, San Jose, CA, USA).

\section{Cytotoxic assay}

Cytotoxicity was determined by 3-(4, 5-dimethylthiazol-2-yl)-2, 5diphenyl tetrazolium bromide (MTT) assay (Roche Diagnostics Corp., GmbH). Logarithmically growing cells were seeded at $3-5 \times 10^{3}$ cells well $^{-1}$ in microtitre plate wells (96 wells, flat bottom) in a final volume of $100 \mu \mathrm{l}$ culture medium per well, in a humidified atmosphere $\left(37^{\circ} \mathrm{C}\right.$ and $\left.5 \% \mathrm{CO}_{2}\right)$. The cells were cultured overnight for adherence and gemcitabine was added at a concentration of $0-1 \mathrm{mg} \mathrm{ml}^{-1}$ into the plates. Gemcitabineinduced cytotoxicity was determined after $48-72 \mathrm{~h}$ of exposure. After incubation, $10 \mu \mathrm{l}$ of MTT labelling reagent (final concentration $0.5 \mathrm{mg} \mathrm{ml}^{-1}$ ) was added to each well. The microtitre plate was incubated for $4 \mathrm{~h}$ in a humidified atmosphere. Solubilisation solution $(100 \mu \mathrm{l})$ was added to each well. The plate was allowed to stand overnight in the incubator in a humidified atmosphere. After checking for complete solubilisation of the purple formazan crystals, the spectrophotometrical absorbance of the samples was measured using a model 550 microplate reader (Bio-Rad Laboratories, CA, USA), at a wavelength of $570 \mathrm{~nm}$ corrected to $655 \mathrm{~nm}$. Each independent experiment was performed three times.

\section{Transfection assays and establishment of a stable CEACAM6-transfected cholangiocarcinoma cell line}

Human CEACAM6 cDNA was generated by RT-PCR and subcloned into pcDNA3.1/Hygro $\left.{ }^{(}\right)(+)$expression vector (Invitrogen Corp., Carlsbad, CA, USA) according to the manufacturer's protocol. To confirm accurate insertion into the frame of the expression vector, sequencing chemistry were performed. Transfection into the cholangiocarcinoma cell line (HuCC-T1) was performed by the Lipofectamine ${ }^{\mathrm{TM}} 2000$ method (Invitrogen Crop., Carlsbad, CA, USA) as described previously (Shibata et al, 1999). Then, stable transfected clone expressing abundant CEACAM6 protein were selected after hygromicine B $\left(600 \mu \mathrm{g} \mathrm{ml}^{-1}\right)$ (Invitrogen Crop., Carlsbad, CA, USA) treatment and used for the subsequent experiments. A mock vector-transfected clone was used for control.

\section{In vitro proliferation assay}

MTT assay Logarithmically growing cells were seeded at $5 \times 10^{3}$ cells well $^{-1}$ in microtiter plate wells (96 wells, flat bottom) in a final volume of $100 \mu \mathrm{l}$ culture medium per well. After $0-96 \mathrm{~h}$ culture, spectrophotometrical absorbance of the samples was 
measured as described above. Each independent experiment was performed three times.

ELISA analysis ELISA was performed using the BrdU ELISA kit (Roche Diagnostic Corp., Tokyo, Japan). Logarithmically growing cells were seeded at $1 \times 10^{4}$ cells well $^{-1}$ in microtitre plate wells (96 wells, flat bottom) in a final volume of $100 \mu$ l culture medium per well. After overnight culture, BrdU was added to a concentration of $10 \mu \mathrm{M}$ into each well and incubated for $5 \mathrm{~h}$. Cells were fixed by FixDenat and anti-BrdU-POD reaction liquid was added. The spectrophotometrical absorbance of the samples was measured using a model 550 microplate reader (Bio-Rad Laboratories, CA, USA), at a wavelength of $450 \mathrm{~nm}$. Each independent experiment was performed three times.

\section{Cell cycle analysis}

Cells $\left(1.0 \times 10^{6}\right)$ were preincubated for $48 \mathrm{~h}$ or $72 \mathrm{~h}$ in serum-free medium at $37^{\circ} \mathrm{C}$ and then were kept in medium with serum $(10 \%$ FBS) for $18 \mathrm{~h}$ at $37^{\circ} \mathrm{C}$. The cells were harvested and fixed in $70 \%$ ethanol at $-20^{\circ} \mathrm{C}$. Then, the cells were washed and resuspended in propidium iodide (PI) staining buffer $\left(5 \mu \mathrm{g} \mathrm{ml}^{-1} \mathrm{PI}\right.$ and $0.25 \mathrm{mg} \mathrm{ml}^{-1}$ RNase) in PBS. DNA content was evaluated using an EPICS XL flow cytometer (Beckman Coulter Corp., Tokyo, Japan).

\section{In vitro invasion assay}

In vitro invasion assays were performed using the $\mathrm{BD}$ BioCoat $^{\mathrm{TM}}$ Tumor Invasion System (Becton Dickinson, San Jose, CA, USA) to evaluate invasive cells as described previously (Albini et al, 1987). Cells $\left(5.0 \times 10^{4}\right.$ cells well $\left.{ }^{-1}\right)$ were placed in the upper chamber, and the lower chamber was filled with $750 \mu \mathrm{l}$ of RPMI1640 with $10 \%$ FBS as a chemoattractant. After $48 \mathrm{~h}$ and $72 \mathrm{~h}$ of incubation at $37^{\circ} \mathrm{C}$, the membranes were labelled with Calcein-AM solutions. The invasive cells that had migrated through the membrane to the lower surface were read in a fluorescence plate reader at excitation/ emission wavelengths of $485 / 530 \mathrm{~nm}$ using Multilabel Plate Counters VICTOR3 (PerkinElmer, Inc., USA).

\section{Anoikis assay}

Anoikis was induced by poly (2-hydroxyethyl methacrylate) (polyHEMA, Sigma) culture. A solution of $120 \mathrm{mg} \mathrm{m}^{-1}$ poly-HEMA in $100 \%$ ethanol was made and diluted $1: 10$ in $95 \%$ ethanol. Of this solution $0.95 \mu \mathrm{l}$ was pipetted into $35 \mathrm{~mm}$ wells and left to dry for $48 \mathrm{~h}$ at room temperature. Prior to use, the wells were washed twice with PBS and once with RPMI-1640. Cells $\left(1 \times 10^{6}\right)$, suspended in $2 \mathrm{ml}$ of RPMI-1640 with $10 \%$ FBS, were incubated in the poly-HEMA-coated wells for $18 \mathrm{~h}$ in a humidified $\left(37^{\circ} \mathrm{C}, 5 \% \mathrm{CO}_{2}\right)$ incubator. Following the induction of anoikis, the cells were washed and resuspended in $0.5 \mathrm{ml}$ of binding buffer, and annexin V/fluorescein isothiocyanate/ propidium iodide labelling was performed in accordance with the manufacturer's protocol (BD biosciences). Analysis was performed by FACScan. A total of 10000 cells were collected for each sample, and the data were analysed with CellQuest software (Becton Dickinson, San Jose, CA, USA).

\section{CEACAM6 RNA interference}

CEACAM6-specific siRNA (Silencer ${ }^{\mathrm{TM}}$ Predesigned siRNA; sense: GGAGGUUCUUCUACUCGCCtt, antisense: GGCGAGUAGA AGAACCUCCtt) and negative control siRNA (Silencer ${ }^{\mathrm{TM}}$ Negative Control siRNA) were purchased from Ambion, USA. Logarithmically growing cells (TFK-1) were seeded at either $1.5 \times 10^{5}$ or $3 \times 10^{3}$ cells well $^{-1}$ in a final volume of $2 \mathrm{ml}$ or $100 \mu \mathrm{l}$ in six- or 96well flat-bottom microtitre plates, respectively. The cells were cultured overnight for adherence. siRNA oligomer was diluted with Opti-MEN $^{\circledR}$ I Reduced Sereum Medium (Invitrogen Corp.,
Carlsbad, CA, USA) and incubated for $5 \mathrm{~min}$ at room temperature. The diluted siRNA oligomer was mixed with the diluted Lipofectamine ${ }^{\mathrm{TM}} 2000$ and incubated for $20 \mathrm{~min}$ at room temperature to allow siRNA-Lipofectamine ${ }^{\mathrm{TM}} 2000$ complexes to form. The siRNA-Lipofectamine ${ }^{\mathrm{TM}} 2000$ complexes were added to each well to a final concentration of $50 \mathrm{pmol} \mathrm{ml}^{-1}$. The cells were incubated in humidified atmosphere $\left(37^{\circ} \mathrm{C}\right.$ and $\left.5 \% \mathrm{CO}_{2}\right)$ and the growth medium was replaced after $4 \mathrm{~h}$. The assay was performed after $48 \mathrm{~h}$ incubation.

\section{Statistical analysis}

For continuous variables, the data were expressed as the means \pm standard deviation (s.d.). The relationship between the CEACAM6 expression and clinicopathological factors and in vitro assay data were analysed using the Student's $t$ test, the Wilcoxon/ Kruskal-Wallis test, $\chi^{2}$ test, and Repeated Measures ANOVA analysis. Overall survival curves and disease-free survival curves were plotted according to the Kaplan-Meier method measured from the day of surgery, and the generalised Wilcoxon test and the log-rank test were applied for comparison. All differences were deemed significant at the level of $P<0.05$. Statistical analysis was performed with the JMP software package (SAS Institute Inc., Cary, NC, USA).

\section{RESULTS}

\section{Clinical study}

Expression of CEACAM6 mRNA in cell lines and clinical tissue specimens Expression of CEACAM6 mRNA in cell lines was shown by RT-PCR. TFK-1 cells showed a higher expression level of CEACAM6 mRNA than that of HuCC-T1 and MEC cells. The expression of CEACAM6 mRNA in cancer and noncancerous tissues of the patients with intrahepatic cholangiocarcinoma was examined by RT - PCR and real-time PCR. The expression levels of CEACAM6 mRNA, which were corrected for those of GAPDH mRNA, in cancer tissues $(5.90 \pm 8.74$; mean \pm s.d.) were higher than in noncancerous tissues $(0.70 \pm 0.48$; mean \pm s.d.) in 16 of the 23 cases $(69.6 \%)$. No differences in mRNA mean expression level was found between cancer and noncancerous tissues statistically $(P=0.10$ : the Wilcoxon/Kruskal-Wallis test) (Figure 1A and B). The cases with values of CEACAM6 under 2 in cancer tissues were considered to be a low expression group $(n=13)$, whereas those with values 2 or over were considered to be a high expression group $(n=10)$. The clinical implications of CEACAM6 in patients with intrahepatic cholangiocarcinoma were evaluated by comparisons between these two groups.

\section{The clinicopathological significance of CEACAM6 mRNA expression in intrahepatic cholangiocarcinoma}

The clinicopathological features analysed in relation to the CEACAM6 expression status are given in Table 1. The clinicopathological factors showed a tendency that the high expression group (seven of $10,70.0 \%$ lymphatic invasion) showed more invasiveness of lymph nodes compared to the low expression group (four of $13,30.8 \%$ lymphatic invasion) $(P=0.06)$. The high expression group (six of $10,60.0 \%$ stage 4 ) was also at a later progression stage of disease than the low expression group (three of $13,23.0 \%$ stage 4$)(P=0.09)$. On the other hand, no significant differences were observed regarding age, gender, tumour size, lymph node metastasis, vascular invasion, perineural invasion or histology. In the disease-free survival curve, patients in the high expression group had a significantly poorer prognosis than those in the low expression group, as illustrated in Figure 1C $(P<0.05$; Wilcoxon test, log-rank test). The overall survival rate between the 
A

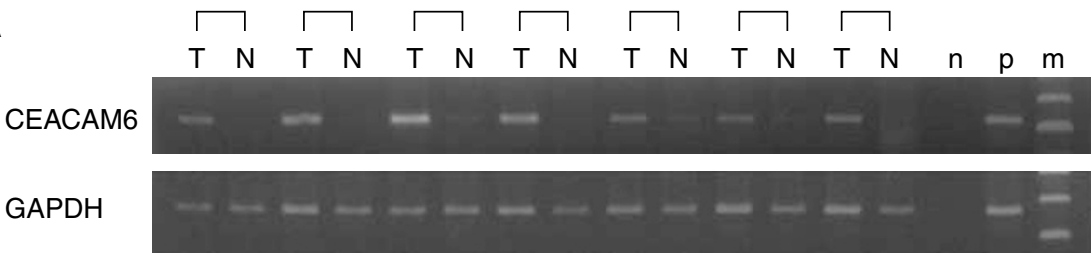

B

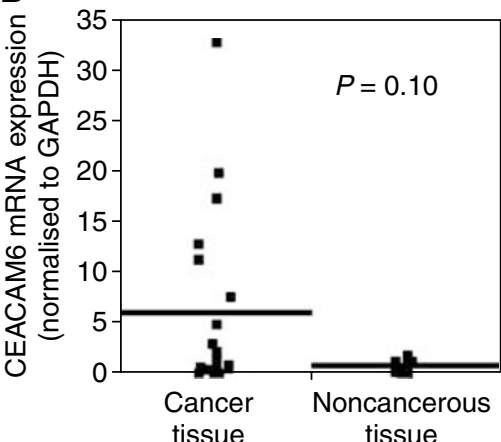

C

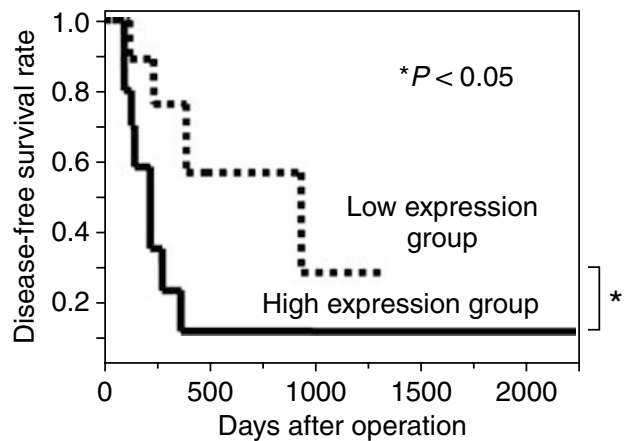

Figure I (A) Expression of CEACAM6 by RT-PCR in representative intrahepatic cholangiocarcinoma patient tissues (T: cancer tissue, N: noncancerous tissue, n: negative control, p: positive control, m: indicates marker). (B) CEACAM6 mRNA expression in cancer and noncancerous tissue with intrahepatic cholangiocarcinoma patients by real-time PCR $(n=23)$. Horizontal lines indicate means. $(\mathbf{C})$ Kaplan-Meier disease-free survival curves in patients with intrahepatic cholangiocarcinoma according to the level of CEACAM6 mRNA expression. The recurrence rate for patients in the high expression group was significantly higher than that for patients in the low expression group $(P<0.05)$. High expression group $(n=10)$ : $C E A C A M 6 / G A P D H \geqslant 2, L O W$ expression group $(n=13)$ : CEACAM6/GAPDH $<2$.

Table I CEACAM6 gene expression and the clinicopathological features of twenty-three patients with intrahepatic cholangiocarcinoma

\section{CEACAM6/GAPDH}

High expression group Low expression group

\begin{tabular}{|c|c|c|c|}
\hline & $\geqslant 2(n=10)$ & $<2(n=13)$ & $P$-value \\
\hline Age & $64.0 \pm 3.9$ & $61.3 \pm 3.5$ & 0.61 \\
\hline \multicolumn{4}{|l|}{ Gender } \\
\hline Male & 7 & 10 & 0.54 \\
\hline Female & 3 & 3 & \\
\hline Tumour size & $6.3 \pm 0.7$ & $5.1 \pm 0.6$ & 0.21 \\
\hline \multicolumn{4}{|c|}{ Lymph node metastasis } \\
\hline Absent & 5 & 10 & 0.18 \\
\hline Present & 5 & 3 & \\
\hline \multicolumn{4}{|c|}{ Lymphatic invasion } \\
\hline Absent & 3 & 9 & 0.06 \\
\hline Present & 7 & 4 & \\
\hline \multicolumn{4}{|c|}{ Vascular invasion } \\
\hline Absent & 2 & 4 & 0.46 \\
\hline Present & 8 & 9 & \\
\hline \multicolumn{4}{|c|}{ Perineural invasion } \\
\hline Absent & 4 & 9 & 0.16 \\
\hline Present & 6 & 4 & \\
\hline \multicolumn{4}{|l|}{ Histology } \\
\hline Moderate & 8 & 6 & 0.11 \\
\hline Poor & 2 & 7 & \\
\hline \multicolumn{4}{|l|}{ Stage } \\
\hline $1,2,3$ & 4 & 10 & 0.09 \\
\hline 4 & 6 & 3 & \\
\hline
\end{tabular}

two groups was not statistically different $(P=0.84$; log-rank test $)$ (data not shown).

\section{Immunohistochemical staining}

Staining of CEACAM6 was markedly stronger in cancer tissue than corresponding noncancerous hepatic tissue. Expression of CEACAM6 was localized to the cell membrane (Figure 2). The immunohistochemical results closely corresponded with the RT - PCR.

\section{Experimental study}

Chemosensitivity of gemcitabine in cholangiocarcinoma cell lines Flow cytometry analysis of expression levels of the CEACAM6 protein in cholangiocarcinoma cell lines showed that TFK-1 cells had higher expression levels of the CEACAM6 protein than the other two cell lines (HuCC-T1, MEC) (Figure 3A). The expression levels of CEACAM6 protein was correlated with that of mRNA. We analysed the relation between CEACAM6 expression and gemcitabine sensitivity in cholangiocarcinoma cell lines. MTT assays for sensitivity of gemcitabine showed TFK-1 cells were more resistant than HuCC-T1 and MEC cells $(P<0.01$ : Repeated Measures ANOVA analysis) (Figure 3B). TFK-1 cells that highly expressed CEACAM6 were more resistant to gemcitabine than other cell lines.

\section{The effect of CEACAM6 gene transfected into the} cholangiocarcinoma cell line

HuCC-T1 cells showed a low expression level of CEACAM6. Stable CEACAM6 overexpressing clones were established using the pcDNA3.1/hygro ${ }^{(1)}(+)$ expression vector. Overexpression of CEACAM6 was confirmed with RT-PCR and flow cytometry (Figure 4A). 

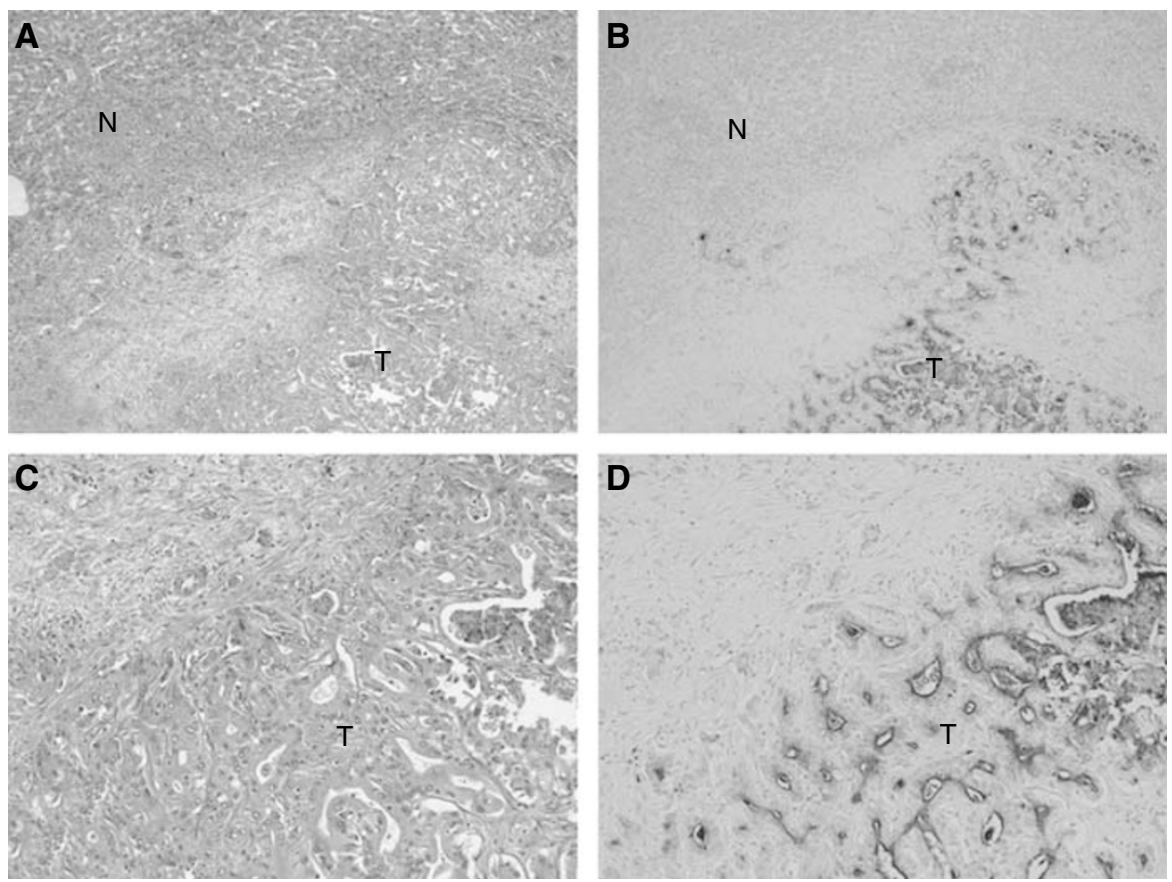

Figure 2 Immunohistochemistry with CEACAM6 antibody in intrahepatic cholangiocarcinoma. The majority of stain was observed in cancer cells. (A) original magnification $\times 40$, haematoxylin and eosin stain, $($ B) original magnification $\times 40$, CEACAM6 stain, $($ C) original magnification $\times 100$, haematoxylin and eosin stain, $(\mathbf{D})$ original magnification $\times 100$, CEACAM6 stain, T: cancer tissue, N: noncancerous tissue.
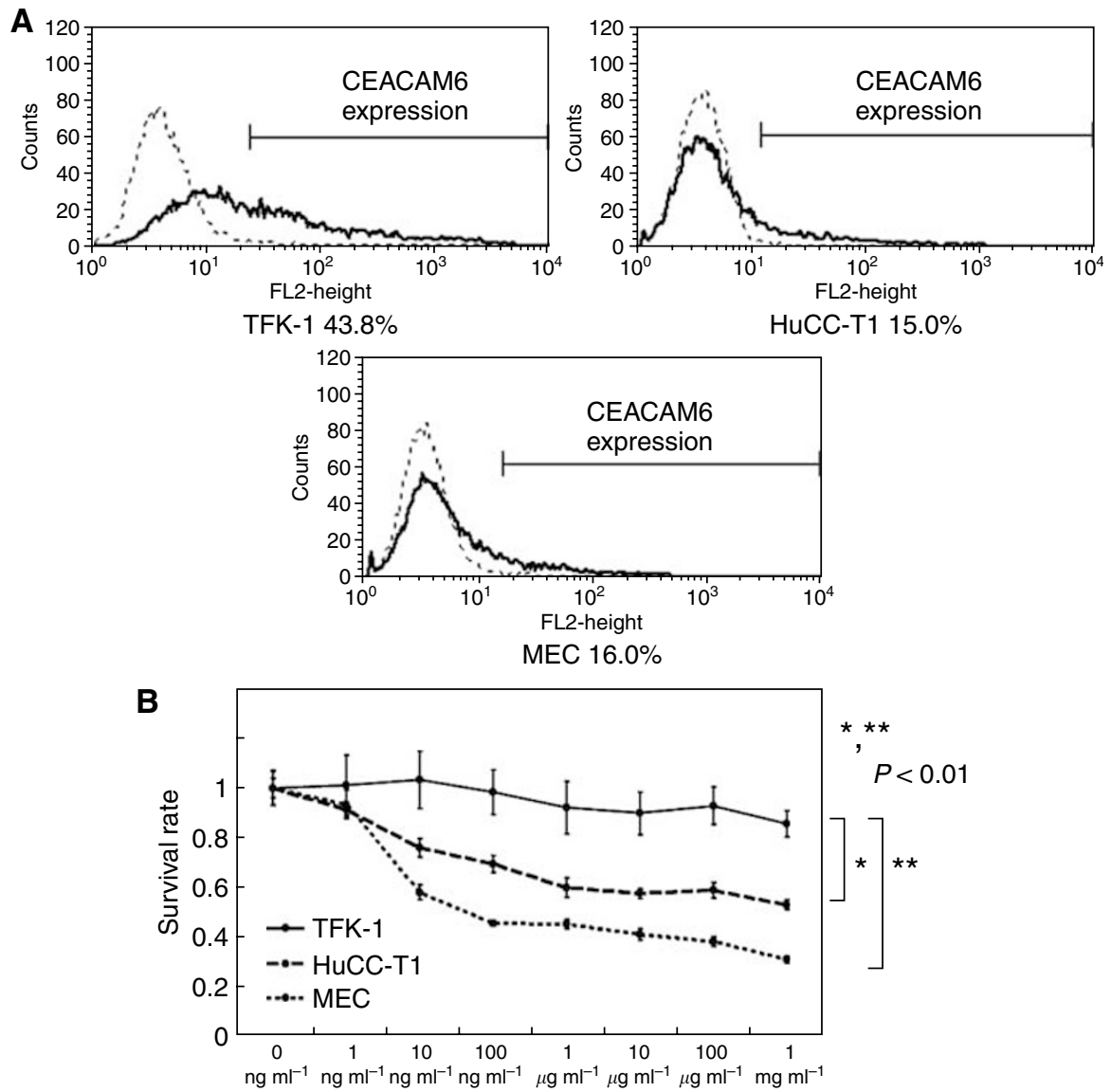

Gemcitabine concentration

Figure 3 (A) Expression of CEACAM6 in three cholangiocarcinoma cell lines (TFK-I, HuCC-TI, MEC) by flow cytometry. TFK-I cells showed higher expression of CEACAM6 than HuCC-TI and MEC. (B) Gemcitabine chemosensitivity of cholangiocellular carcinoma cell lines. After $48 \mathrm{~h}$ gemcitabine exposure, survival rates of each cells were measured by MTT assay. TFK-I cells were more chemoresistant than HuCC-TI and MEC $(P<0.0 \mathrm{I})$. The data represent the mean \pm s.d. 


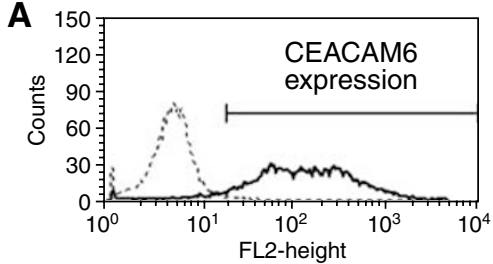

C

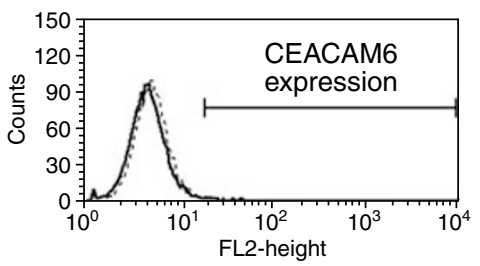

CEACAM6 transfectant $95.1 \%$

Mock

$0.5 \%$

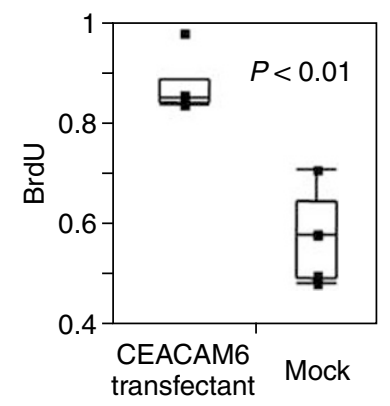

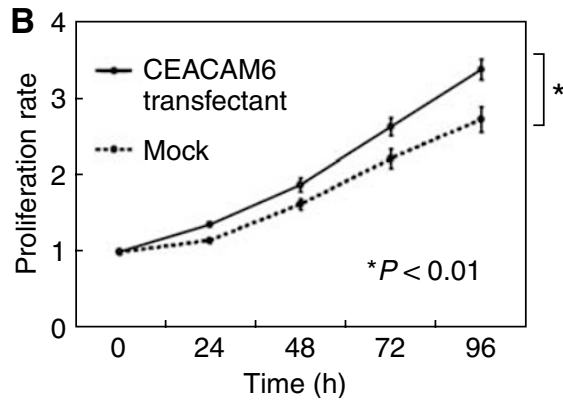

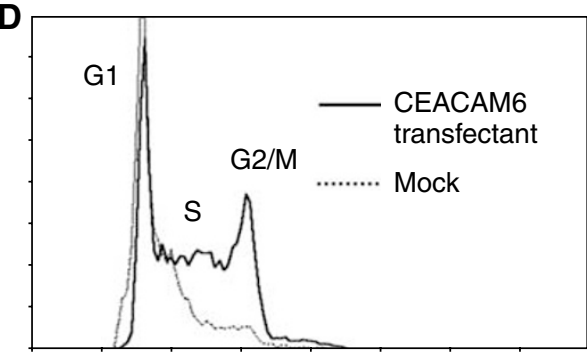

Figure 4 (A) CEACAM6 Expression of transfectants and mock cells (HuCC-TI). Expression levels of CEACAM6 protein were markedly increased in transfectants. (B) MTT assay. CEACAM6-transfectants were more proliferative than mock cells $(P<0.0 \mathrm{I})$. The data represent the mean + s.d. (C) Comparison of BrdU uptake. The average BrdU uptake of transfectants was increased compared with mock cells. The data represent the mean \pm s.d. (D) Cell cycle analysis of transfectants and mock cells. After $48 \mathrm{~h}$ starvation and I8 $\mathrm{h}$ incubation with serum, transfectants (73.1\%) were more in $\mathrm{S}$ phase than mock cells (63.4\%)

We analysed whether overexpression of CEACAM6 would alter the growth rate of HuCC-T1 cholangiocarcinoma cells. As shown in Figure $4 \mathrm{~B}$ and $4 \mathrm{C}$, there was a significant difference in growth rate between the CEACAM6 overexpressing cells and the mocktransfected cells (MTT assay: $P<0.01$; Repeated Measures ANOVA analysis, ELISA analysis: $P<0.01$; Student's $t$ test). In cell cycle analysis, after $48 \mathrm{~h}$ serum starvation and $18 \mathrm{~h}$ incubation with serum, a greater percentage of CEACAM6 overexpressing cells $(73.1 \% \mathrm{~S}$ phase) were in $\mathrm{S}$ phase than mock-transfected cells (63.4\% S phase) (Figure 4D). After $72 \mathrm{~h}$ serum starvation, the same pattern was identified.

In the clinicopathological studies, we found a tendency that the incidence of lymphatic invasion was higher in the CEACAM6 high expression group than in the CEACAM6 low expression group (Table 1). To verify these findings in an in vitro assay, we examined the invasive potential of the CEACAM6 overexpressing cells using an in vitro matrigel invasion assay. Invasion assay showed that CEACAM6 overexpressing cells were more significantly invasive than mock-transfected cells $(P<0.01$ : the Wilcoxon/ Kruskal-Wallis test) (Figure 5A). High expression of CEACAM6 enhanced tumour invasiveness.

Anoikis is associated with cellular invasion and metastatic potential. After anoikis-induced culture, mock-transfected cells (16.7\%) were more apoptotic than CEACAM6 overexpressing cells (10.6\%). CEACAM6 overexpressing cells were more resistant to anoikis than mock-transfected cells (Figure $5 \mathrm{~B}$ ).

We examined whether CEACAM6 expression in cholangiocarcinoma cell line (HuCC-T1) would alter sensitivity to gemcitabine. We compared CEACAM6 overexpressing cells and mocktransfected cells. Overexpression of CEACAM6 induced chemoresistance to gemcitabine which was significantly increased as observed by the MTT assay $(P<0.01$ : Repeated Measures ANOVA analysis) (Figure 5C).

\section{The effect of CEACAM6 gene silencing on gemcitabine chemosensitivity}

TFK-1 cells showed a high expression level of CEACAM6 and strong chemoresistance to gemcitabine. We examined whether suppression of CEACAM6 expression would alter sensitivity to gemcitabine. The expression level of mRNA and protein was suppressed by CEACAM6-specific siRNA as confirmed by RT-PCR and flow cytometry analysis (Figure 6A). Suppression of CEACAM6 expression reduced chemoresistance to gemcitabine $(P<0.01$ : Repeated Measures ANOVA analysis $)$ (Figure 6B).

\section{DISCUSSION}

CEACAM6 is highly expressed in various human cancer tissues and its clinical significance has been widely reported (Hasegawa et al, 1993; Kodera et al, 1993; Scholzel et al, 2000; Ilantzis et al, 2002; Jantscheff et al, 2003; Duxbury et al, 2004a). CEACAM6 overexpression independently predicts poor overall and diseasefree survival, and correlates inversely with cellular differentiation in colorectal cancer (Ilantzis et al, 2002; Jantscheff et al, 2003). Duxbury et al (2005) showed that CEACAM6 expression was associated with adverse pathologic features and prognosis in pancreatic cancer. In gastric cancer, CEACAM6 was overexpressed; however, there was no significant association between the expression level and clinicopathological features (Yasui et al, 2004). This study of intrahepatic cholangiocarcinoma indicates that higher expression of CEACAM6 correlated with lymph node invasion $(P=0.06)$, advanced stage of the disease $(P=0.09)$, and poorer prognosis $(P<0.05)$. Thus, its association between CEACAM6 expression and poor prognosis in intrahepatic 

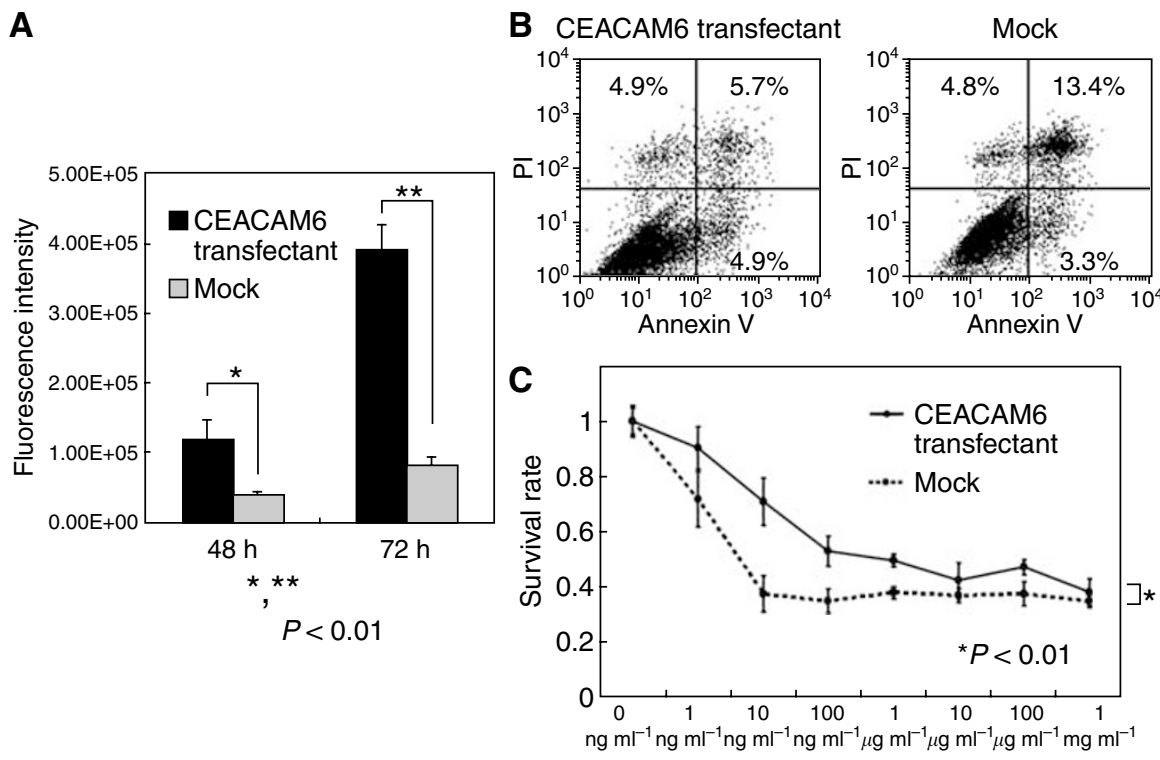

Gemcitabine concentration

Figure 5 (A) Invasion assay. The invasiveness of transfectants was significantly stronger than that of mock cells $(P<0.0 \mathrm{I})$. The data represent the mean \pm s.d. (B) Anoikis analysis. After anoikis induction for $8 \mathrm{~h}$, the apoptosis rates were measured by Annexin $\mathrm{V}$ and $\mathrm{Pl}$ staining. Apoptosis cells were calculated as UR + LR (transfectant: UL: 4.9\%, UR: 5.7\%, LL: 84.5\%, LR: 4.9\%, mock: UL: 4.8\%, UR: I3.4\%, LL: 78.5\%, LR: 3.3\%). Proportion of apoptotic cells in transfectants (10.6\%) was less than mock cells (16.7\%). (UL: upper left, UR: upper right, LL: lower left, LR: lower right) (C) Gemcitabine chemosensitivity of transfectants and mock cells. After $72 \mathrm{~h}$ of gemcitabine exposure, survival rates were measured by MTT assay. Transfectants were more chemoresistant than mock cells $(P<0.0 \mathrm{I})$. The data represent the mean \pm s.d.
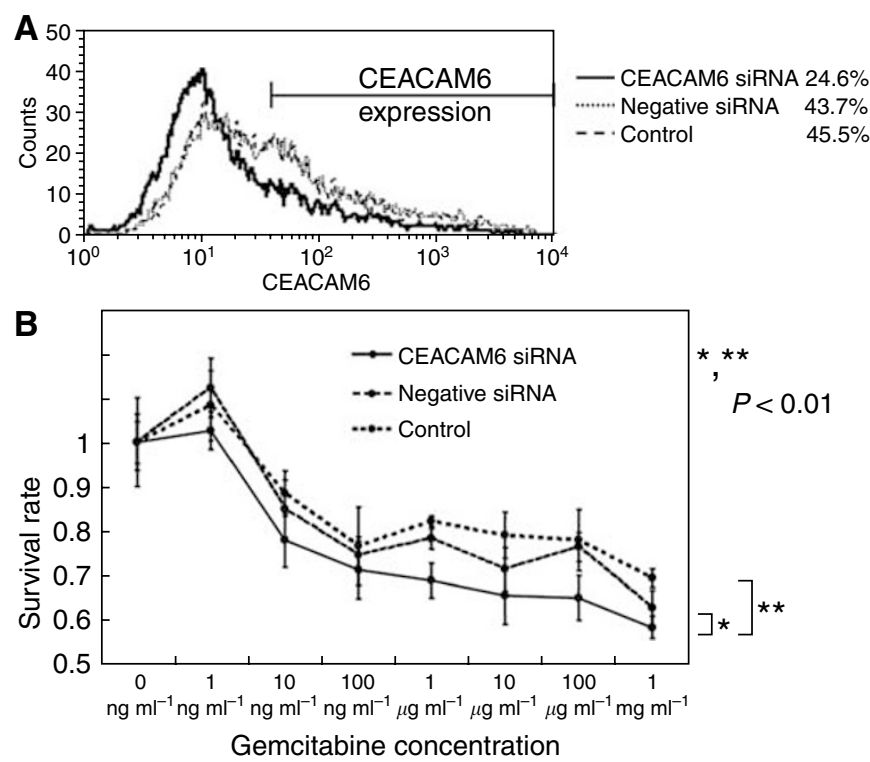

Figure 6 (A) CEACAM6 Expression of CEACAM6 suppressed cells by CEACAM6 specific-siRNA and control cells (TFK-I). $48 \mathrm{~h}$ after siRNA addition, expression of CEACAM6 was measured by flow cytometry. (B) Gemcitabine chemosensitivity of CEACAM6 suppressed cells and control cells. $72 \mathrm{~h}$ after gemcitabine exposure, survival rates were measured by MTT assay. CEACAM6 suppressed cells were more chemosensitive to gemcitabine than mock cells $(P<0.0 \mathrm{I})$. The data represent the mean \pm s.d.

cholangiocarcinoma is similar to that in colorectal cancer or pancreatic cancer.

The reason why CEACAM6 overexpression is associated with aggressive biological behaviour of cancer cells has not been fully clarified. However, to date several possibilities have been considered. Overexpression of CEACAM6 is associated with greater resistance to anoikis (Ordonez et al, 2000; Ilantzis et al, 2002), a subset of apoptosis induced by inadequate or inappropriate cell substrate adhesion, and increased Akt and c-Src kinase activities (Duxbury et al, 2004a). Resistance to anoikis is a property of transformed cells that is associated with greater cellular invasive ability and in vivo metastatic potential (Yawata et al, 1998; Shanmugathasan and Jothy, 2000). Wandering CEACAM6 overexpressed cells that are resistant to anoikis can reside in the liver and contribute to recurrence. Increased invasiveness following CEACAM6 overexpression is associated with upregulation of IGF-IR expression and MMP2 expression, which depends upon Akt activation induced by CEACAM6 overexpression (Duxbury et al, 2004c). c-Src-dependent modulation of MMP9 activity contributes significantly to the increased cellular invasiveness induced by CEACAM6 overexpression (Duxbury et al, 2004d). This study demonstrates that the transfectants of the CEACAM6 gene were more proliferative in MTT assay (Figure 4B and C), had greater invasive abilities in matrigel assay (Figure 5A) and were more resistant to anoikis in anoikis assay (Figure 5B) compared to mock-transfected cells, concurring with the reports described above. Therefore, in intrahepatic cholangiocarcinoma clinical data, these results would be associated with lymphatic invasion and poor prognosis. Furthermore, CEACAM6 gene silencing reversed the acquired anoikis resistance and inhibited metastatic ability (Duxbury et al, 2004a). Suppression of CEACAM6 expression by siRNA impairs pancreatic adenocarcinoma xenograft growth in vivo and improves the survival of tumour-bearing nude mice (Duxbury et al, 2004b). Blocking the $\mathrm{N}$ and A1B1 domains of CEACAM5/CEACAM6 can impede the metastatic process (Blumenthal et al, 2005). Interestingly, another study showed that the CEACAM6 gene was one of the most overexpressed genes in a side population of cells of hepatocellular carcinoma (Haraguchi et al, 2006). Most of the side population of cells are considered to be equal to stem cells that show strong chemoresistance to anticancer drugs. Targeting CEACAM6 may conceivably be a therapeutic approach for patients with cholangiocarcinoma. 
It is difficult to treat cholangiocarcinoma. One reason is that most patients have advanced disease far beyond surgical treatment. The other is that the effects of chemotherapy for cholangiocarcinoma are largely disappointing (Kelley et al, 2004; Olnes and Erlich, 2004; Khan et al, 2005). 5-Fluorouracil (5-FU) as a single therapy or in combination with other drugs has been studied extensively for cholangiocarcinoma. Although many of these trials were small and uncontrolled, there was an overall response rate of $0-40 \%$ and median survival of 2-12 months. With respect to gemcitabine, response rates of $8-60 \%$ and median overall survival of 6.3-16 months have been reported. These rates were not changed even when gemcitabine was used in combination with other agents (Thongprasert, 2005). Our study within cholangiocarcinoma cell lines demonstrated that CEACAM6 overexpressed cells were more chemoresistant to gemcitabine than mock-transfected cells and suppression of CEACAM6 expression by CEACAM6 siRNA increased chemosensitivity to gemcitabine. One possible explanation of the relationship between overexpression of CEACAM6 and gemcitabine resistance is that CEACAM6 overexpression may protect cells from cytochrome c-induced caspase 3 activation and apoptosis via Akt or c-Src activation. This cytoprotective pathway may contribute to gemcitabine chemoresistance (Duxbury et al, 2004e).

\section{REFERENCES}

Albini A, Iwamoto Y, Kleinman HK, Martin GR, Aaronson SA, Kozlowski JM, McEwan RN (1987) A rapid in vitro assay for quantitating the invasive potential of tumor cells. Cancer Res 47: $3239-3245$

Blumenthal RD, Hansen HJ, Goldenberg DM (2005) Inhibition of adhesion, invasion, and metastasis by antibodies targeting CEACAM6 (NCA-90) and CEACAM5 (Carcinoembryonic Antigen). Cancer Res 65: $8809-8817$

Duxbury MS, Ito H, Zinner MJ, Ashley SW, Whang EE (2004a) CEACAM6 gene silencing impairs anoikis resistance and in vivo metastatic ability of pancreatic adenocarcinoma cells. Oncogene 23: 465-473

Duxbury MS, Matros E, Ito H, Zinner MJ, Ashley SW, Whang EE (2004b) Systemic siRNA-mediated gene silencing: a new approach to targeted therapy of cancer. Ann Surg 240: 667-674, discussion 675-6

Duxbury MS, Ito H, Benoit E, Zinner MJ, Ashley SW, Whang EE (2004c) Overexpression of CEACAM6 promotes insulin-like growth factor Iinduced pancreatic adenocarcinoma cellular invasiveness. Oncogene 23: $5834-5842$

Duxbury MS, Ito H, Benoit E, Ashley SW, Whang EE (2004d) CEACAM6 is a determinant of pancreatic adenocarcinoma cellular invasiveness. $\mathrm{Br} \mathrm{J}$ Cancer 91: 1384-1390

Duxbury MS, Ito $\mathrm{H}$, Benoit $\mathrm{E}$, Waseem $\mathrm{T}$, Ashley SW, Whang EE (2004e) A novel role for carcinoembryonic antigen-related cell adhesion molecule 6 as a determinant of gemcitabine chemoresistance in pancreatic adenocarcinoma cells. Cancer Res 64: $3987-3993$

Duxbury MS, Matros E, Clancy T, Bailey G, Doff M, Zinner MJ, Ashley SW, Maitra A, Redston M, Whang EE (2005) CEACAM6 is a novel biomarker in pancreatic adenocarcinoma and PanIN lesions. Ann Surg 241: $491-496$

Haraguchi N, Utsunomiya T, Inoue H, Tanaka F, Mimori K, Barnard GF, Mori M (2006) Characterization of a side population of cancer cells from human gastrointestinal system. Stem Cells 24: 506-513

Hasegawa T, Isobe K, Tsuchiya Y, Oikawa S, Nakazato H, Nakashima I, Shimokata K (1993) Nonspecific crossreacting antigen (NCA) is a major member of the carcinoembryonic antigen (CEA)-related gene family expressed in lung cancer. $\mathrm{Br} J$ Cancer 67: 58-65

Ilantzis C, DeMarte L, Screaton RA, Stanners CP (2002) Deregulated expression of the human tumor marker CEA and CEA family member CEACAM6 disrupts tissue architecture and blocks colonocyte differentiation. Neoplasia 4: 151-163

Jantscheff P, Terracciano L, Lowy A, Glatz-Krieger K, Grunert F, Micheel B, Brummer J, Laffer U, Metzger U, Herrmann R,
In conclusion, this study provided important information that overexpression of CEACAM6 expression may become a prognostic marker and a chemoresistant indicator for gemcitabine in patients with cholangiocarcinoma. CEACAM6 could play an important role in cholangiocarcinoma. Further studies in greater numbers of patients should lead to a final conclusion.

\section{ACKNOWLEDGEMENTS}

We thank $\mathrm{T}$ Shimooka, K Ogata, M Oda, M Kasagi, and Y Nakagawa for their technical assistance. This work was supported in part by the following grants and foundations: a Grant-in-Aid for Scientific Research on Priority Areas of Cancer (14026037, 15023245) and Grants-in-Aid for development of far advanced medicine (B) (2002-2005), from the Ministry of Education, Culture, Sports, Science and Technology of Japan, a Grant-in-Aid for Scientific Research (S) (17109013), (B) (14370358, 15390398, $15390379,16390381)$ and (C) (14570149, 14571140, 15591412, 15591411), YASUDA Medical Research Foundation, Sankyo Foundation of Life Science, and a Grant-in-Aid for Exploratory Research $(14657286,16659337,1665960)$ from the Japan Society for Promotion of Science.
Rochlitz C (2003) Expression of CEACAM6 in resectable colorectal cancer: a factor of independent prognostic significance. J Clin Oncol 21: $3638-3646$

Kelley ST, Bloomston M, Serafini F, Carey LC, Karl RC, Zervos E, Goldin S, Rosemurgy P, Rosemurgy AS (2004) Cholangiocarcinoma: advocate an aggressive operative approach with adjuvant chemotherapy. Am Surg 70: $743-748$, discussion $748-749$

Khan SA, Davidson BR, Goldin R, Pereira SP, Rosenberg WM, Taylor-Robinson SD, Thillainayagam AV, Thomas HC, Thursz MR, Wasan H (2002) Guidelines for the diagnosis and treatment of cholangiocarcinoma: consensus document. Gut 1(Suppl 6): VI1 - VI9

Khan SA, Thomas HC, Davidson BR, Taylor-Robinson SD (2005) Cholangiocarcinoma. Lancet 366: $1303-1314$

Kodera Y, Isobe K, Yamauchi M, Satta T, Hasegawa T, Oikawa S, Kondoh K, Akiyama S, Itoh K, Nakashima I et al (1993) Expression of carcinoembryonic antigen (CEA) and nonspecific crossreacting antigen (NCA) in gastrointestinal cancer; the correlation with degree of differentiation. $\mathrm{Br}$ J Cancer 68: 130 - 136

Lazaridis KN, Gores GJ (2005) Cholangiocarcinoma. Gastroenterology 128: $1655-1667$

Mori M, Mimori K, Yoshikawa Y, Shibuta K, Utsunomiya T, Sadanaga N, Tanaka F, Matsuyama A, Inoue H, Sugimachi K (2002) Analysis of the gene-expression profile regarding the progression of human gastric carcinoma. Surgery 131: S39-47

Ogawa K, Utsunomiya T, Mimori K, Tanaka F, Inoue H, Nagahara $H$, Murayama S, Mori M (2005) Clinical significance of human kallikrein gene 6 messenger RNA expression in colorectal cancer. Clin Cancer Res 11: $2889-2893$

Olnes MJ, Erlich R (2004) A review and update on cholangiocarcinoma. Oncology 66: $167-179$

Ordonez C, Screaton RA, Ilantzis C, Stanners CP (2000) Human carcinoembryonic antigen functions as a general inhibitor of anoikis. Cancer Res 60: 3419-3424

Scholzel S, Zimmermann W, Schwarzkopf G, Grunert F, Rogaczewski B, Thompson J (2000) Carcinoembryonic antigen family members CEACAM6 and CEACAM7 are differentially expressed in normal tissues and oppositely deregulated in hyperplastic colorectal polyps and early adenomas. Am J Pathol 156: 595-605

Shaib Y, El-Serag HB (2004) The epidemiology of cholangiocarcinoma. Semin Liver Dis 24: $115-125$

Shanmugathasan M, Jothy S (2000) Apoptosis, anoikis and their relevance to the pathobiology of colon cancer. Pathol Int 50: 273-279 
Shibata K, Tanaka S, Shiraishi T, Kitano S, Mori M (1999) G-protein gamma 7 is down-regulated in cancers and associated with p 27kip1induced growth arrest. Cancer Res 59: 1096-1101

Thompson JA, Grunert F, Zimmermann W (1991) Carcinoembryonic antigen gene family: molecular biology and clinical perspectives. J Clin Lab Anal 5: $344-366$

Thongprasert S (2005) The role of chemotherapy in cholangiocarcinoma. Ann Oncol Suppl 2: ii93 - ii96
Yasui W, Oue N, Ito R, Kuraoka K, Nakayama H (2004) Search for new biomarkers of gastric cancer through serial analysis of gene expression and its clinical implications. Cancer Sci 95: $385-392$

Yawata A, Adachi M, Okuda H, Naishiro Y, Takamura T, Hareyama M, Takayama S, Reed JC, Imai K (1998) Prolonged cell survival enhances peritoneal dissemination of gastric cancer cells. Oncogene 16: $2681-2686$ 